\title{
Un enfoque de optimización para el control de semáforos
}

\author{
Dominguez Patricia Neri \\ Dto. de Ingeniería, Universidad Nacional del Sur, Centro de Investigaciones en Mecánica \\ Teórica y Aplicada Facultad Regional Bahía Blanca Universidad Tecnológica Nacional. \\ Argentina
}

\begin{abstract}
Cortínez Víctor Hugo
Centro de Investigaciones en Mecánica Teórica y Aplicada Facultad Regional Bahía Blanca Universidad Tecnológica Nacional, Dto. de Ingeniería, Universidad Nacional del Sur, Consejo Nacional de Investigaciones Científicas y Técnicas. Argentina.
\end{abstract}

\section{RESUMEN}

En este trabajo se presenta un modelo computacional para el diseño óptimo de configuración de semáforos basado en la combinación del modelo de tráfico LQM (Link Queue Model) desarrollado recientemente por Wen-Long Jin (2013) con una técnica de optimización denominada Recocido Simulado.

El modelo LQM conduce a un sistema de ecuaciones diferenciales ordinarias no lineales de primer orden teniendo tantas incógnitas (densidades medias espaciales) como tramos existan en la red. Se ha adoptado el mencionado modelo de tráfico ya que ha demostrado una gran efectividad balanceando adecuadamente la velocidad computacional, la estabilidad numérica y el realismo físico de sus predicciones.

Por otra parte, se utiliza el método de Recocido Simulado como técnica de optimización ya que el problema analizado es no convexo, es decir la función objetivo puede presentar varios mínimos (o máximos) locales.

Se presentan ejemplos numéricos que ilustran las posibilidades de la presente metodología.

\section{INTRODUCCIÓN}

El control de tráfico por medio de la semaforización se ha convertido en un elemento fundamental para aliviar la congestión en las grandes ciudades considerando la falta de espacio para la construcción de nuevas arterias.

Un aspecto fundamental para efectuar el diseño del control de tráfico es la predicción de los patrones de flujo vehicular en la red de transporte para la infraestructura existente y sus posibles modificaciones ante diversos parámetros del funcionamiento de los semáforos. Las variables más importantes para realizar tal diseño corresponden a los tiempos de duración del ciclo así como también de la señal verde. También juega un papel de importancia la sincronización del comienzo de ciclo en diferentes intersecciones sucesivas. Luego, el problema de diseño consiste en obtener estos parámetros para las diferentes señales de tráfico de manera tal de mejorar la eficiencia del transporte con algún objetivo previamente definido.

Se han empleado diferentes maneras de evaluar tal eficiencia. Una de ellas consiste en la consideración de la cantidad total de vehículos en una zona urbana o equivalentemente de la 
longitud de las colas de espera formadas en cada arteria. Otra forma consiste en la medición del flujo vehicular en las arterias bajo análisis. Se pretende buscar las condiciones que minimicen la primera forma de la función objetivo y/o maximicen la segunda forma en un período determinado.

Existen diversos enfoques que permiten el análisis dinámico del comportamiento de los vehículos en la red de tráfico. Una posible clasificación de tales metodologías consiste en un enfoque microscópico que considera en forma independiente cada uno de los vehículos y otro macroscópico que tiene en cuenta el comportamiento dinámico de agregados de vehículos tales como densidades y flujos en los tramos de la red. Estos últimos modelos en general se basan en una analogía con fenómenos hidrodinámicos en tuberías o canales, por tal motivo se los denomina teorías hidrodinámicas de tráfico.

Entre los modelos que describen el tráfico desde el punto de vista microscópico pueden citarse aquellos desarrollados por Herman et al. (1959), Gipps (1986), Nagel y Schreckenberg (1992). Este tipo de enfoque microscópico se ha utilizado frecuentemente para el análisis de áreas limitadas de una ciudad tales como 2 ó 3 intersecciones simultáneas. Sin embargo, cuando se pretende analizar áreas grandes de una red, o eventualmente la red completa, la modelización de la cinemática de cada uno de los vehículos puede ser una tarea imposible o al menos muy costosa desde el punto de vista computacional. En tales casos puede ser ventajoso utilizar los modelos hidrodinámicos de tráfico urbano.

Estos modelos se basan en la teoría de Lighthill y Whitham (1955) y Richards (1956) o teoría LWR, que describe el comportamiento de ondas cinemáticas en un medio continuo. Este tipo de modelo viene gobernado mediante ecuaciones diferenciales a derivadas parciales hiperbólicas. Asimismo, se han estudiado diversas formas para modelar el comportamiento en intersecciones con caminos convergentes o divergentes (Coclite el al., 2005) utilizando tal tipo de teoría.

Recientemente, se ha desarrollado un modelo de optimización de semáforos en redes de tráfico combinando un modelo LWR con un enfoque numérico de optimización del tipo entero-mixto lineal (Göttlich et al., 2015). Esta metodología es muy precisa aunque puede ser muy costosa desde el punto de vista computacional para grandes redes.

A los efectos de aliviar la carga computacional se han diseñado simplificaciones del modelo LWR tales como el método CTM (Cell Transmission Model) desarrollado por Daganzo (1994, 1995), que es una aproximación de diferencias finitas al LWR que permite obtener resultados similares a aquel con menor tiempo de cálculo.

Lo et al. (2001) han desarrollado una formulación de control de semáforos (DISCO) basada en la combinación del modelo CTM con el método de optimización de Algoritmos Genéticos. La metodología DISCO ha mostrado ser eficiente en diversos escenarios brindando resultados comparables y aún mejores que el código comercial TRANSYT (Courage y Wallace, 1991).

Un enfoque interesante para optimizar la semaforización en una red urbana consiste en la utilización del modelo de Gazis y Potter (1963). Esta formulación es muy conveniente por su simplicidad y describe los efectos de las señales de tráfico sobre el promedio temporal de los flujos atravesando una intersección. Tal simplicidad lo hace apto para el estudio de 
control de semáforos en tiempo real (Mayorano el al., 2007). Sin embargo, pasa por alto diversos fenómenos de importancia en el comportamiento del tráfico urbano tal como la dinámica de parada y arranque o la formación o disipación de embotellamientos.

Un enfoque alternativo que modela la dinámica en los tramos y en las intersecciones considerando aspectos de importancia tales como paradas y arranques fue desarrollado recientemente por Jin (2013) y se denomina modelo LQM (Link Queue Model). Representa un avance frente a otros modelos basados en tramos tales como el de Abu-Lebdeh y Banchoal (1997), ya que se utiliza el diagrama fundamental, de manera modificada, a los efectos de calcular los flujos máximos de salida o recepción en cada tramo. A su vez éstos son modificados para definir los flujos entre un tramo y otro considerando condiciones de continuidad y de interacción entre diferentes tramos en intersecciones convergentes y/o divergentes. Las variables fundamentales de tal enfoque vienen dadas por las densidades promediadas espacialmente en cada tramo y conduce a un sistema de tantas ecuaciones diferenciales ordinarias como tramos existan en la red.

Jin (2013) ha demostrado que tal enfoque constituye una aproximación continua al modelo de onda cinemática sobre una red de tráfico, siendo aún más económica que el CTM permitiendo capturar diversos fenómenos de importancia tales como iniciación, propagación y disipación de colas.

En este trabajo se propone un enfoque para la optimización off-line del control de semáforos basado en una combinación del modelo LQM con una técnica de optimización estocástica denominada Recocido Simulado. El problema consiste en seleccionar los parámetros de la semaforización de manera tal de maximizar el flujo medio temporal en una zona determinada de la red de tráfico.

Se ha adoptado el modelo LQM por el balance entre realismo y velocidad computacional ya que esta última cualidad facilita su aplicación en un procedimiento que requiere numerosos cálculos cambiando las variables de diseño. Asimismo, para minimizar la cantidad de evaluaciones de la función objetivo (FO) y automatizar la búsqueda hacia los valores óptimos se propone la utilización del método de Recocido Simulado ya que se trata de una técnica estocástica de búsqueda global que evita la convergencia hacia máximos locales. Este hecho es de gran importancia ya que la FO es no convexa. Esta técnica ha sido utilizada en diversas áreas de la ingeniería y en particular en problemas de tráfico urbano (Dominguez y Cortínez, 2012)

En la sección 2 se presentan los fundamentos del modelo LQM, en la sección 3 se formula el problema de optimización de los parámetros del sistema de semáforos, en la sección 4 se presenta el método numérico de resolución conjuntamente con las particularidades de la técnica de Recocido Simulado, en la sección 5 se dan algunos ejemplos numéricos y finalmente, en la sección 6 se exponen las conclusiones y futuros trabajos.

\section{MODELO DE TRÁFICO (LQM)}

Se describen brevemente los fundamentos de la teoría de flujo de tráfico en redes de transporte propuesta por W-L Jin (2013). Las variables fundamentales en este enfoque 
corresponden a las densidades vehiculares $k_{a}$ correspondientes a cada tramo $a$ de la red. Para un tiempo dado, a partir del conocimiento de las densidades es posible determinar los flujos de entrada $f_{a}$ y de salida $g_{a}$ de cada uno de los tramos.

La dinámica del sistema viene gobernada por la conservación de tráfico en cada uno de los tramos que componen la red, es decir:

$$
\frac{\partial k_{a}(t)}{\partial t}=\frac{1}{L_{a}}\left(f_{a}-g_{a}\right)
$$

donde $L_{a}$ corresponde a la longitud de cada tramo.

Los flujos de entrada y salida de cada tramo dependen de las densidades en el sistema en un momento dado debiendo verificar las leyes de tráfico en el interior de los tramos, la continuidad de vehículos en las intersecciones y la compatibilidad con las posibilidades de almacenamiento. Dentro de cada uno de los tramos el flujo vehicular debe contemplar la ley de Greenshields (1934), es decir, las relaciones posibles empíricamente definidas entre flujo y densidad e indicadas en el diagrama fundamental de tráfico.

Al utilizar la teoría LWR la relación fundamental aplica a cada elemento de longitud de un camino. En el presente modelo se efectúa la simplificación consistente en la constancia de la densidad sobre la longitud de cada tramo, consecuentemente, la aplicación del diagrama fundamental debe ser modificada definiendo los flujos de entrada y salida en función de un solo valor de densidad. De esta manera, se define la demanda $d_{a}$ y la oferta $s_{a}$ como los flujos máximos posibles de egreso e ingreso a cada arco respectivamente de acuerdo a una modificación del diagrama fundamental. Las correspondientes expresiones son las siguientes:

$$
\begin{aligned}
& d_{a}(t)=Q_{a}\left(\min \left\{k_{a}(t), k_{a, c}(t)\right\}\right)=\left\{\begin{array}{cc}
Q_{a}\left(k_{a}(t)\right) & k_{a}(t) \in\left\lfloor 0, k_{a, c}\right\rfloor \\
C_{a} & k_{a}(t) \in\left[k_{a, c}, k_{a, j}\right]
\end{array}\right. \\
& s_{a}(t)=Q_{a}\left(\min \left\{k_{a}(t), k_{a, c}(t)\right\}\right)=\left\{\begin{array}{cc}
C_{a} & k_{a}(t) \in\left\lfloor 0, k_{a, c}\right\rfloor \\
Q_{a}\left(k_{a}(t)\right) & k_{a}(t) \in\left[k_{a, c}, k_{a, j}\right]
\end{array}\right.
\end{aligned}
$$

En estas expresiones $C_{a}$ es la capacidad del tramo y $k_{a, c}$ y $k_{a, j}$ son la densidades crítica y de embotellamiento respectivamente de cada tramo. Tales magnitudes pueden apreciarse en el diagrama de la Figura 1. 


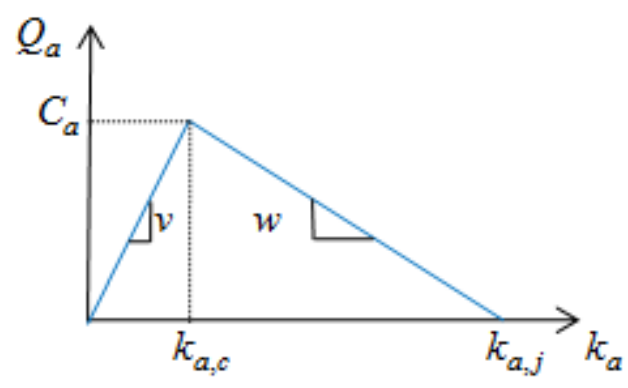

\section{Figura 1 - Diagrama fundamental del tráfico vehicular}

La posibilidad real de que se den tales flujos dependerá de la interacción entre los tramos en las intersecciones. A continuación se expresan las formulas que determinan los flujos entrantes y salientes en cada tramo de acuerdo a su estado como así también atendiendo a la conservación de vehículos en cada intersección. En este trabajo se consideran los tipos de intersecciones que se muestran en la Figura 2.

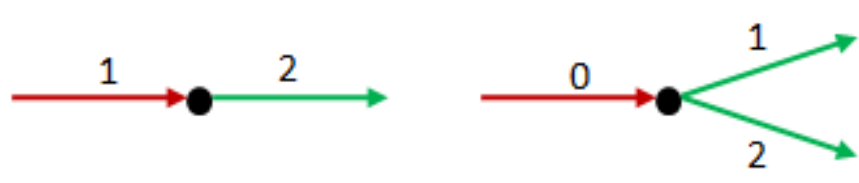

Lineal

Divergencia

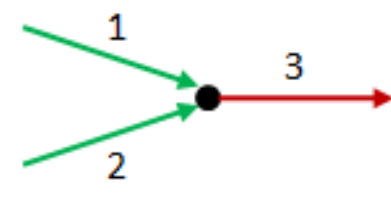

Encuentro

Figura 2 - Tipos de intersecciones

Unión lineal

$$
g_{1}(t)=f_{2}(t)=\pi(t) \min \left\{d_{1}(t), s_{2}(t)\right\}
$$

Divergencia

$$
\begin{aligned}
g_{0}(t)=\pi(t) \min & \left\{d_{0}(t), \frac{s_{1}(t)}{\xi_{0 \rightarrow 1}(t)}, \frac{s_{2}(t)}{\xi_{0 \rightarrow 2}(t)}\right\} \\
f_{1}(t) & =\xi_{0 \rightarrow 1}(t) g_{0}(t) \\
f_{2}(t) & =\xi_{0 \rightarrow 2}(t) g_{0}(t)
\end{aligned}
$$

donde $\xi_{0 \rightarrow 1}(t)$ y $\xi_{0 \rightarrow 2}(t)$ son las proporciones de vehículos del tramo 0 dirigiéndose hacia los tramos 1 y 2 respectivamente. Observar que debe cumplirse que tales cantidades son positivas y que suma debe ser igual a 1. En este trabajo se supone que tales proporciones son fijas dependiendo de elecciones de rutas predefinidas. 
Encuentro

$$
\begin{gathered}
f_{3}(t)=\min \left\{d_{1}(t)+d_{2}(t), s_{3}(t)\right\} \\
g_{1}(t)=\min \left\{d_{1}(t), \max \left\{s_{3}(t)-d_{2}(t), \frac{C_{1}}{C_{1}+C_{2}} s_{3}(t)\right\}\right\} \\
g_{2}(t)=f_{3}(t)-g_{1}(t)
\end{gathered}
$$

Donde $C_{1}$ y $C_{2}$ corresponden a las capacidades de los tramos 1 y 2 respectivamente. Se ha introducido en la formulas de intersección la variable $\pi(t)$ que describe la permeabilidad de la salida del tramo debida a la existencia de un semáforo:

$$
\pi(t)= \begin{cases}1 & \text { sem. verde } \\ 0 & \text { sem. rojo }\end{cases}
$$

El anterior, constituye un sistema de $N$ ecuaciones diferenciales ordinarias no lineales, siendo $N$ el número total de tramos de la red. Tal sistema debe ser modificado para las condiciones de ingreso y egreso a la red considerada.

En el trabajo de Jin (2013) pueden encontrarse mayores detalles teóricos así como estudios numéricos que muestran su adecuado poder predictivo.

\section{OPTIMIZACIÓN DE LOS PARÁMETROS DEL SISTEMA DE SEMÁFOROS.}

Las variables de diseño del problema considerado vienen dadas por los ciclos $C_{s}$ de semáforos, tiempos de verde $V_{s}$ y sincronización medida a través de los tiempos de iniciación de los ciclos de los semáforos relativos a uno de ellos $\delta_{s}$, donde $s$ identifica cada unos de los semáforos analizados $\left(s=1,2, \ldots N_{s}\right)$. Todas las variables de diseño se engloban en el arreglo TV.

En este trabajo, se evalúa la eficiencia de la red a partir del flujo total de vehículos en un período determinado, es decir, se pretende que en tal período fluya la mayor cantidad de vehículos posible. Para ello se considera la suma de los flujos de salida de cada uno de los tramos, promediados en un intervalo de tiempo que abarque varios ciclos de semáforo una vez establecido un estado estacionario (o cuasi estacionario). Luego, el problema de optimización se formula de la siguiente manera:

donde

$$
\mathbf{T V}^{\text {opt }}=\arg \text { máx }(F O)
$$

$$
F O=\int_{t_{1}}^{t_{2}} \sum_{a} g_{a} d t
$$

s.a

$$
\mathbf{T V}^{\text {min }} \leq \mathbf{T V} \leq \mathbf{T V}^{\text {máx }}
$$


$\mathbf{T V}^{\text {min }}$ y $\mathbf{T V}^{\text {máx }}$ corresponden a los valores mínimos y máximos que pueden adoptar las variables de diseño.

\section{MODELO NUMÉRICO}

El modelo LQM puede ser resuelto con cualquier método numérico de valor inicial (Euler, Runge-Kutta, etc.), luego, es posible realizar diversos cálculos del sistema aludido para diferentes valores de las variables de diseño comparando numéricamente las correspondientes FO. Sin embargo, tal procedimiento de prueba y error puede requerir grandes tiempos computacionales, en particular para el análisis de grandes redes con apreciable cantidad de semáforos. Por tal motivo es necesario adoptar una metodología de optimización que realice de manera automática la búsqueda de los parámetros que optimice la FO.

Cuando el problema de optimización consiste en resolver una FO no convexa tal como la (8), un modo de evitar que la búsqueda de soluciones finalice en óptimos locales es permitir que algunos movimientos sean hacia soluciones peores. Pero si la búsqueda está avanzando realmente hacia una buena solución, estos movimientos de escape de óptimos locales deben realizarse de un modo controlado. En el método de Recocido Simulado esto se logra controlando la frecuencia de los movimientos de escape mediante una función que hace disminuir la probabilidad de estos movimientos hacia soluciones peores conforme avanza la búsqueda. Se aplica así la filosofía habitual de búsqueda de diversificar al principio e intensificar al final.

Recocido Simulado o "Simulated annealing" deriva su nombre de un proceso termodinámico de "recocido" aplicado a los metales. Tal proceso comprende el calentamiento del metal hasta una alta temperatura definida, el mantenimiento a esa temperatura por suficiente tiempo y luego el enfriamiento a velocidades mínimas, con el fin de mejorar sus propiedades físicas y mecánicas. Un esquema controlado de disminución de la temperatura evita defectos en el metal.

Análogamente, en la técnica de optimización (maximización en este trabajo), el algoritmo comienza con una solución inicial factible y genera a partir de ella una nueva solución en el entorno de la misma. Si la solución vecina es mejor que la actual, es decir aumenta el valor de la FO se acepta automáticamente. Si no, aún existe la posibilidad de que dicha solución sustituya a la solución actual de acuerdo a un cierto criterio probabilístico $e^{-\triangle F O / T}$ que depende de la diferencia entre la solución actual y la nueva ( $\triangle F O=F O$ nueva $-F O$ anterior) y del parámetro $T$ conocido como temperatura. A medida que el algoritmo avanza se va disminuyendo $T$ de manera tal de disminuir la probabilidad de aceptar soluciones factibles que no produzcan una mejora en la función objetivo. El algoritmo acepta soluciones mucho peores que la actual al principio de la ejecución (exploración) pero no al final (explotación). Si la temperatura decrece muy lentamente, el proceso converge a una solución cercana a la óptima. En la Figura 3 se detalla el algoritmo de aplicación del método de Recocido Simulado al diseño óptimo del funcionamiento de semáforos. 
En este trabajo se utilizó el entorno Matlab para implementar el algoritmo de optimización descripto involucrando la resolución de las ecuaciones diferenciales ordinarias del problema de semaforización utilizando la función ODE45.

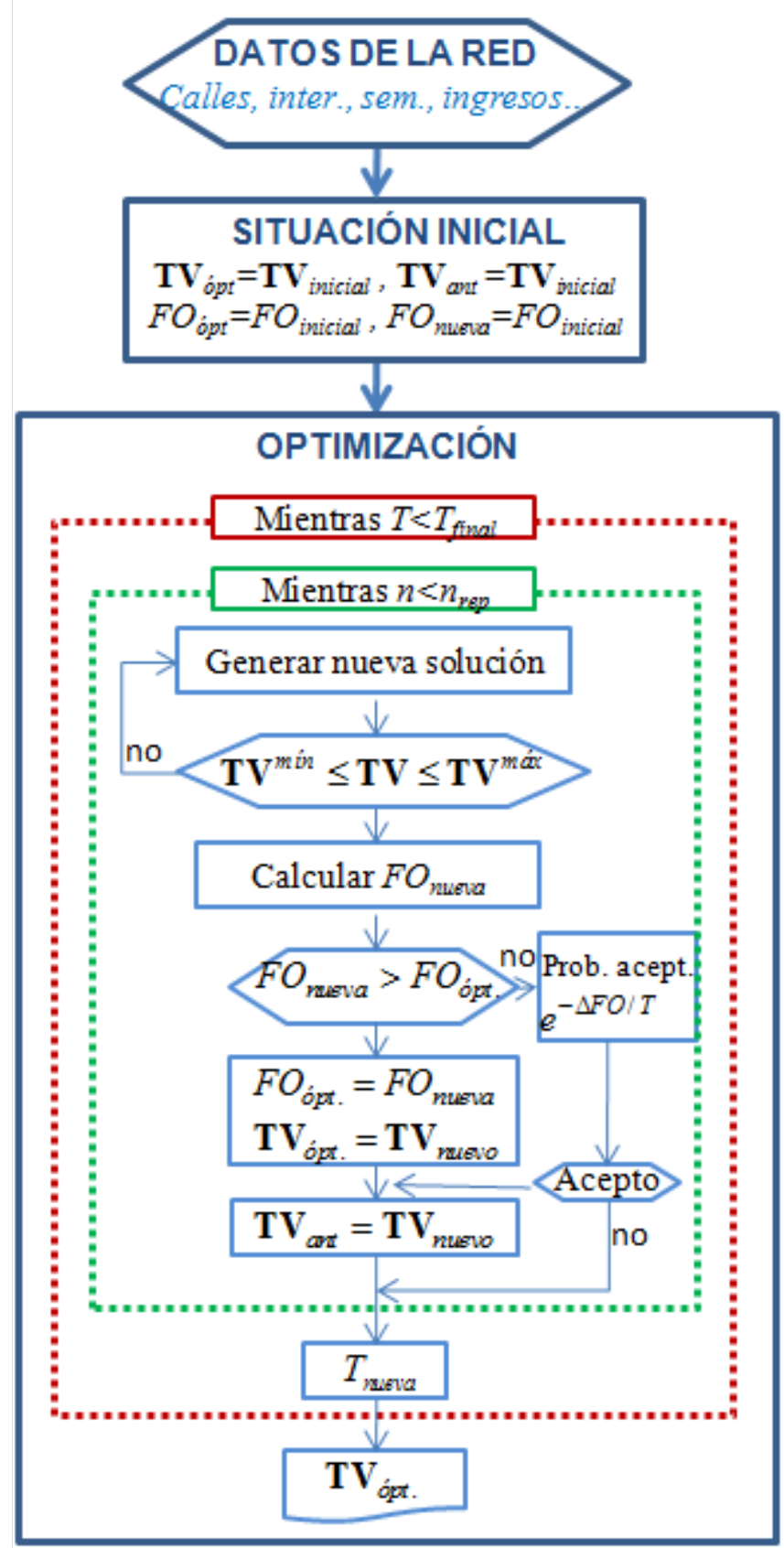

\section{Figura 3 - Método de optimización}

\section{EJEMPLOS NUMÉRICOS}

Se analizan 5 cuadras de una calle donde las direcciones de circulación y las posibilidades de giro se muestran en la Figura 4. La longitud de todas las cuadras es $L_{a}=100 \mathrm{~m}$ y la 
capacidad de cada una de ellas es $C_{a}=1200 \mathrm{veh} / \mathrm{h}$. Se consideran los siguientes valores para los flujos entrantes: $d_{1}=1400, d_{7}=1200, d_{10}=1600, d_{11}=1600 d_{14}=800$ y para los salientes: $s_{9}=1200, s_{13}=1200$ y $s_{15}=800 \mathrm{veh} / \mathrm{h}$ respectivamente, mientras que se adoptan las siguientes tasas de giro: $\xi_{2 \rightarrow 9}=\xi_{4 \rightarrow 13}=0,2$ y $\xi_{7 \rightarrow 2}=\xi_{10 \rightarrow 3}=\xi_{11 \rightarrow 4}=0,5$. Se adoptan asimismo los siguientes valores de velocidad para el diagrama fundamental (Figura 1) $v=40$ y $w=16$ $\mathrm{km} / \mathrm{h}$, quedando determinadas la densidad de congestión $k_{a, c}=30 \mathrm{veh} / \mathrm{km}$ y la densidad de embotellamiento $k_{a, j}=105 \mathrm{veh} / \mathrm{km}$. En todos los casos se considera una densidad inicial $k_{0}=[40,30,30,30,10] \mathrm{veh} / \mathrm{km}$. Todas las intersecciones (A a E) del tramo analizado tienen semáforos de ciclo $C=40$ segundos y en principio todos los ciclos comienzan en el mismo instante.

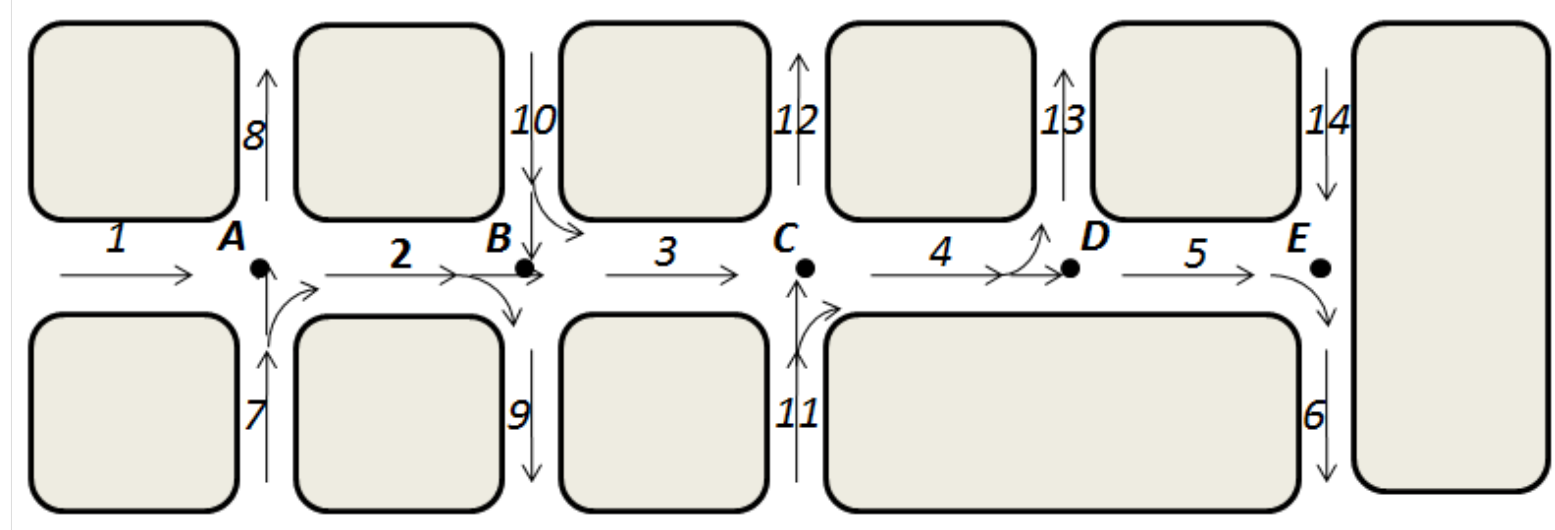

\section{Figura 4 - Esquema de la red de calles.}

En la Figura 5 se puede apreciar la evolución temporal de las densidades de las calles 1 a 5 desde la situación inicial y durante 30 minutos para una configuración cualquiera de tiempos de verde (Tabla 1, configuración b). Como se puede apreciar la densidad es muy elevada en todos los tramos, llegando en los tres primeros muy cerca de la densidad de embotellamiento. En la Figura 6 se observa la variación temporal de la suma de los flujos salientes de los tramos 1 a 5, 7, 10,11 y 14, que corresponden a los utilizados en el proceso de optimización para definir la función objetivo, durante el mismo intervalo de tiempo.

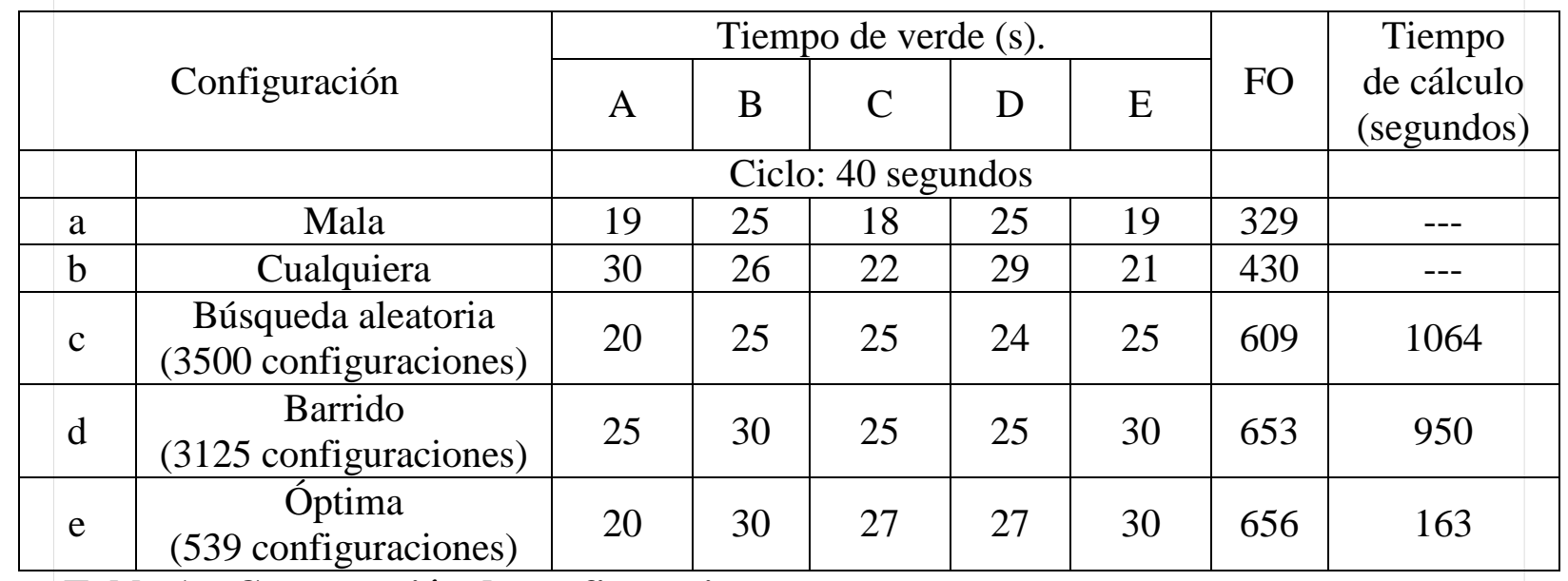

Tabla 1 - Comparación de configuraciones. 


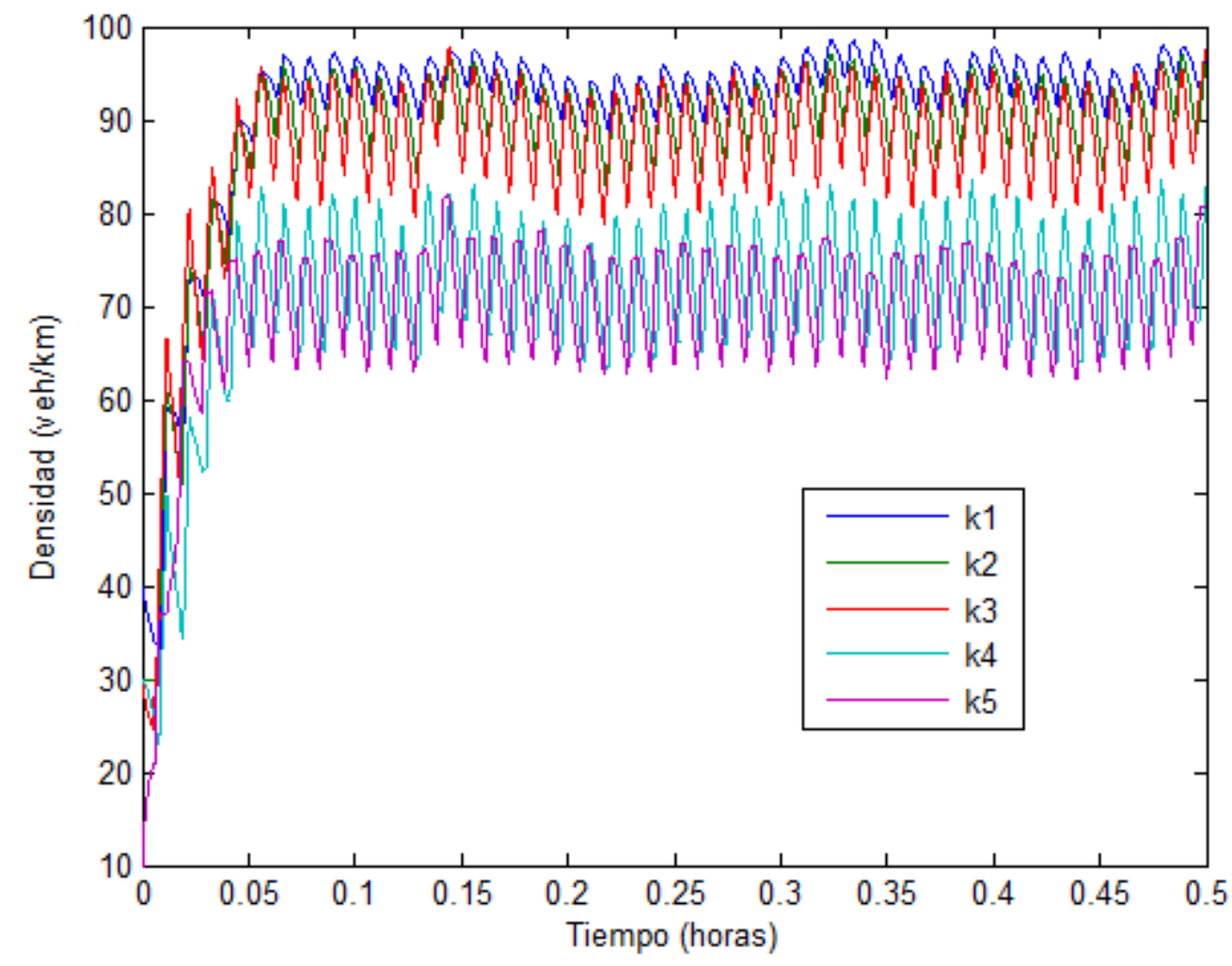

Figura 5 - Densidad en cada cuadra para la configuración b).

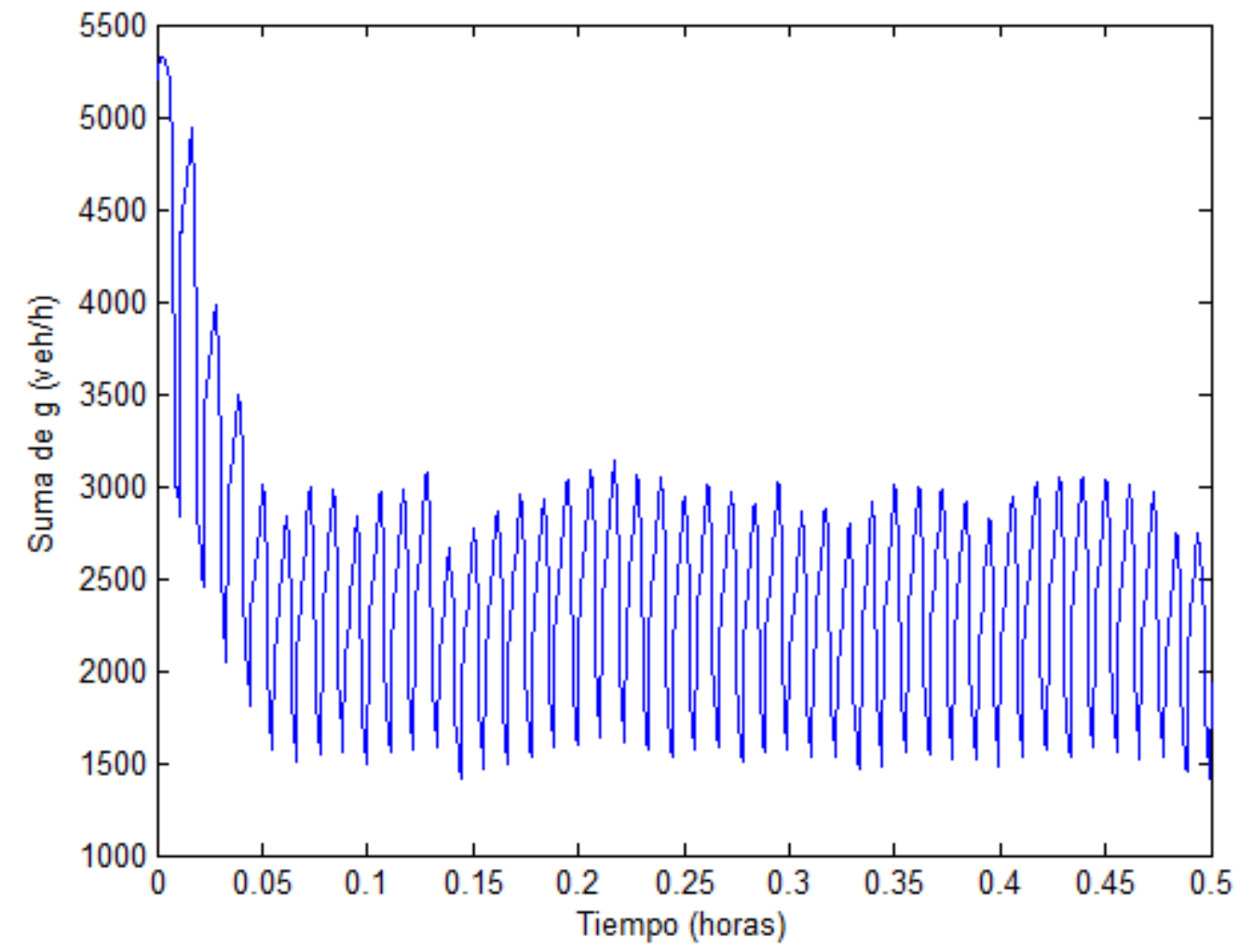

Figura 6 - Suma de flujos salientes de tramos 1 a7, 10, 11 y 14. Configuración b). 
Se intentó a continuación obtener una configuración de tiempos de verde de los semáforos más eficiente en la evacuación de vehículos en la zona. Para ello se seleccionaron los tramos 1 a $5,7,10,11$ y 14 para evaluar la función objetivo de la ecuación (8) y en todos los semáforos se adoptaron tiempos de verde mínimos de 10 y máximos de 30 segundos (ecuación 9) para garantizar el flujo longitudinal sobre la avenida y los correspondientes desplazamientos en las calles transversales. La integral de la FO se evaluó entre 10 y 20 minutos.

Se seleccionaron 3 metodologías (c, d y e en Tabla 1) para generar los valores de las variables de cada configuración. La primera consistió en obtener las nuevas soluciones variando los tiempos de verde de 2 semáforos, elegidos en forma aleatoria, en \pm 1 segundo, también aleatoriamente, con respecto a sus valores previos. En la Tabla 1 se muestran los resultados de la mejor solución obtenida luego de correr 5 búsquedas de 700 alternativas cada una.

En la Figura 7 se muestra la evolución de las soluciones para las 700 alternativas de tal corrida. Como se puede observar la fluctuación de los valores de la función objetivo es muy grande y solo 2 veces aparecen buenos resultados. La segunda forma fue realizar un barrido sistemático de las 5 variables utilizando intervalos de 5 segundos en los tiempos de verde para cada una. Los resultados de la aplicación de este método corresponden a la configuración d) de la Tabla 1.

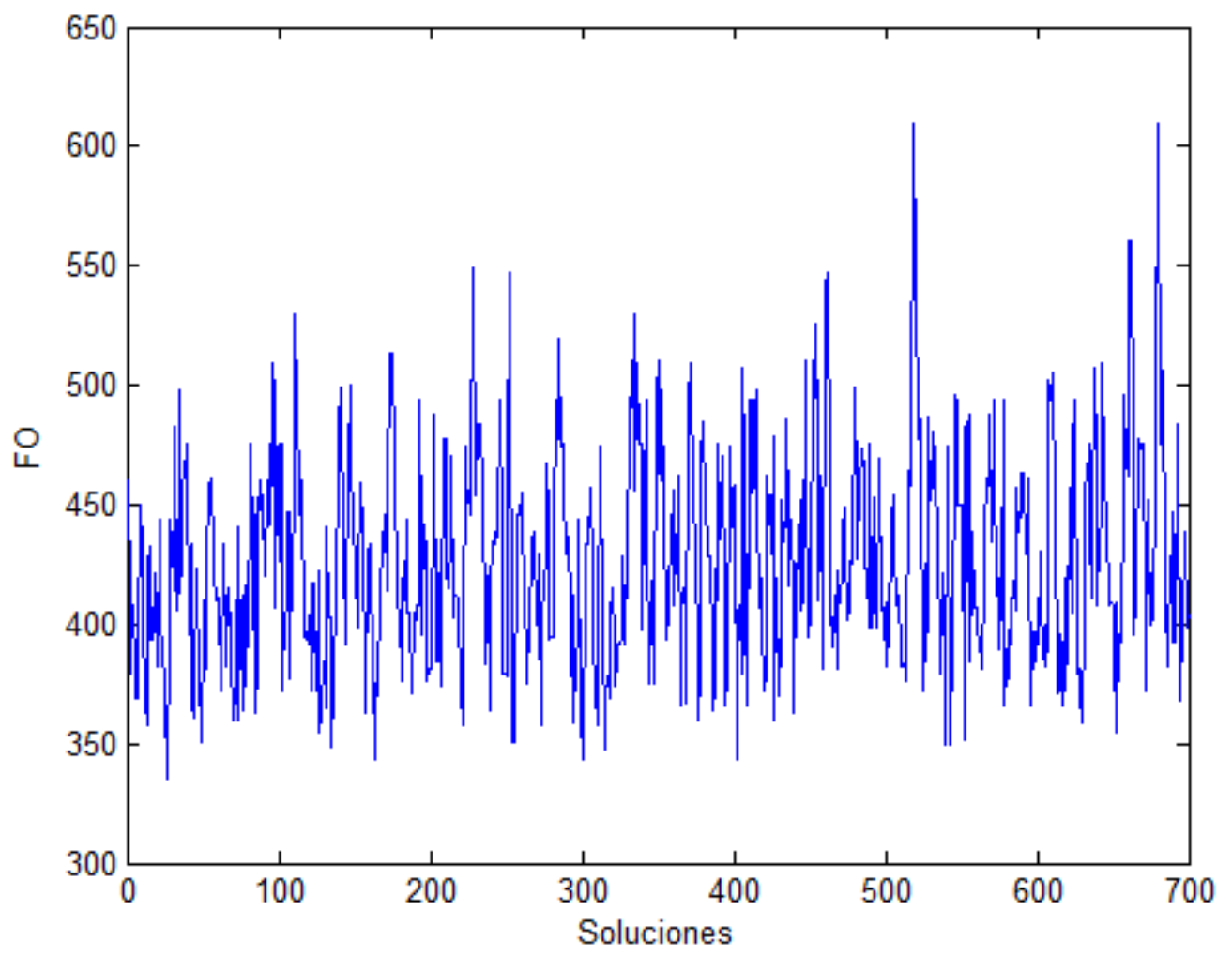

Figura 7 - Soluciones de la FO obtenidas mediante una búsqueda.

Como se puede apreciar la solución es mejor que la anterior, pero el costo computacional aumenta tanto con la cantidad de variables (cantidad de semáforos) como con la cantidad de intervalos considerados. Finalmente, se utilizó una optimización realizada con la técnica de Recocido Simulado mencionado en la sección 4. Los parámetros utilizados en este caso fueron $T_{i}=10^{4}$, cantidad de iteraciones en cada temperatura $n_{\text {rep }}=30$, condición de parada $T=T_{i}^{*} 10^{-3}$ y soluciones no mejores durante 5 cambios de temperatura a partir del décimo cambio. La solución óptima se muestra en la Tabla 1 y fue obtenida en 163 segundos. En la 
Figura 8 se puede apreciar la evolución del algoritmo hacia la solución óptima. En este caso la dirección de la evolución hacia la solución final es claramente apreciable.

Es interesante observar en la Tabla 1 que las configuraciones óptimas o cuasi óptimas pueden duplicar la eficiencia con respecto a la peor configuración (a) y aumentar un $50 \%$ con respecto a una configuración tomada arbitrariamente (b).

Finalmente, en las figuras 9 y 10 se muestran, para la solución óptima, las densidades de las calles y la función utilizada en la definición de la FO, respectivamente. Si se comparan tales evoluciones con las correspondientes a un caso cualquiera (configuración b), excepto para el primer tramo las densidades disminuyen de manera apreciable. Asimismo la suma de los flujos salientes óptimos aumenta considerablemente con respecto a una configuración arbitraria como la mostrada en la Figura 6.

Sobre la misma red se han desarrollado además optimizaciones de semáforos contemplando ciclos y sincronización no obteniéndose cambios apreciables.

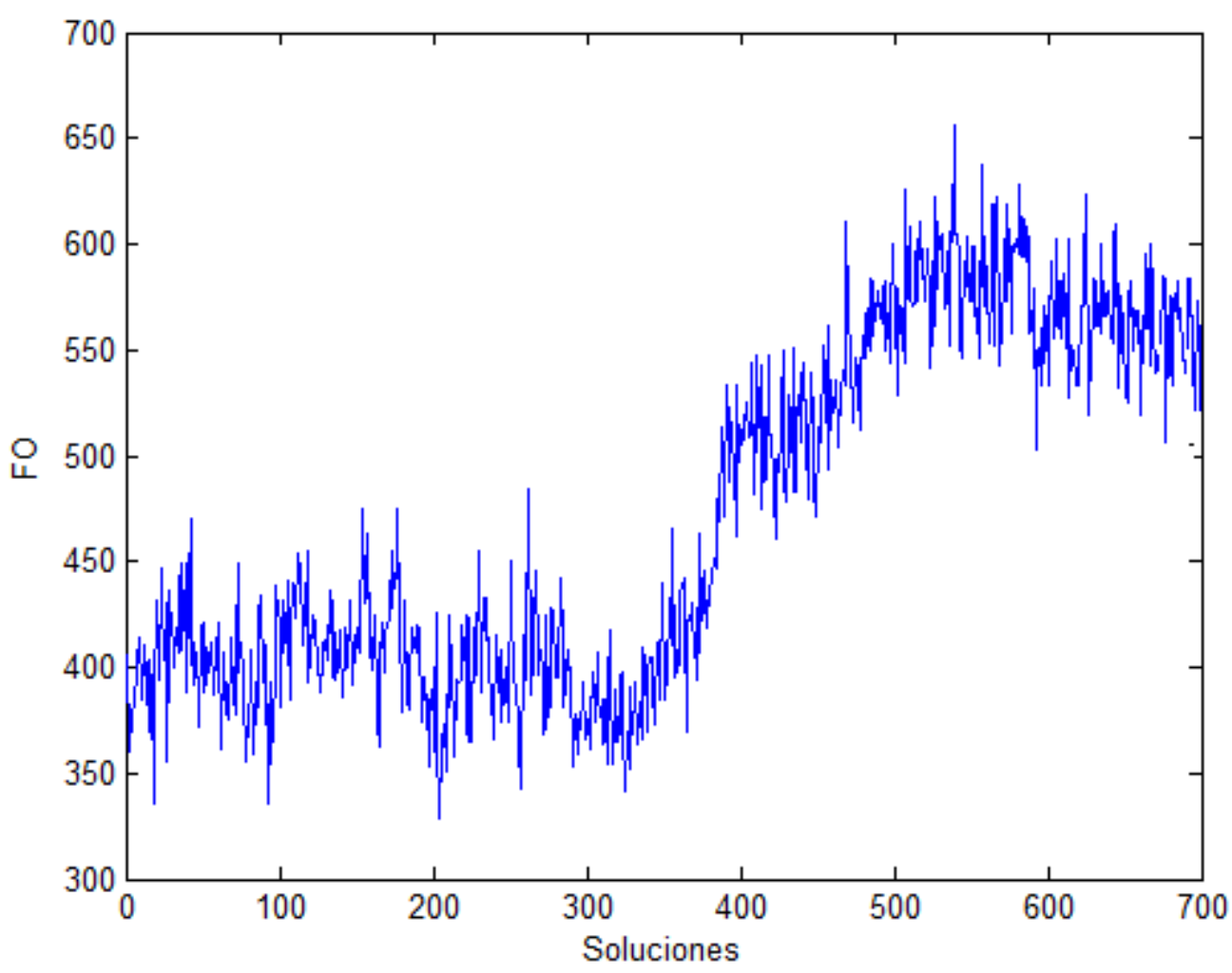

Figura 8 - Soluciones de la FO obtenidas mediante el método de Recocido Simulado.

\section{CONCLUSIONES}

Se ha desarrollado un método para la determinación óptima de los parámetros de semaforización en forma off-line. Tal metodología puede ser de utilidad para la configuración del control de tráfico en momentos del día preestablecidos. El enfoque combina un modelo de tráfico recientemente desarrollado por Jin (2013, ) que balancea poder de predicción con tratabilidad computacional, con un método de optimización aleatorio denominado Recocido Simulado adecuado para la búsqueda de soluciones en problemas con muchos extremos locales como el presentado aquí. El enfoque fue implementado en 
ambiente Matlab.

Se ha presentado un ejemplo numérico muy simple que pone de manifiesto las posibilidades de tal metodología.

Actualmente se está desarrollando un estudio más exhaustivo contemplando redes de mayor complejidad y evaluando la eficiencia computacional respecto a otros métodos pertinentes.

\section{AGRADECIMIENTOS}

El presente trabajo es parte del proyecto "Modelos Matemáticos en Ingeniería Ambiental: Diseño óptimo y control" (Secyt, Universidad Tecnológica Nacional) y del proyecto "Problemas de optimización en Ingeniería Civil" (Departamento de Ingeniería, Universidad Nacional del Sur).

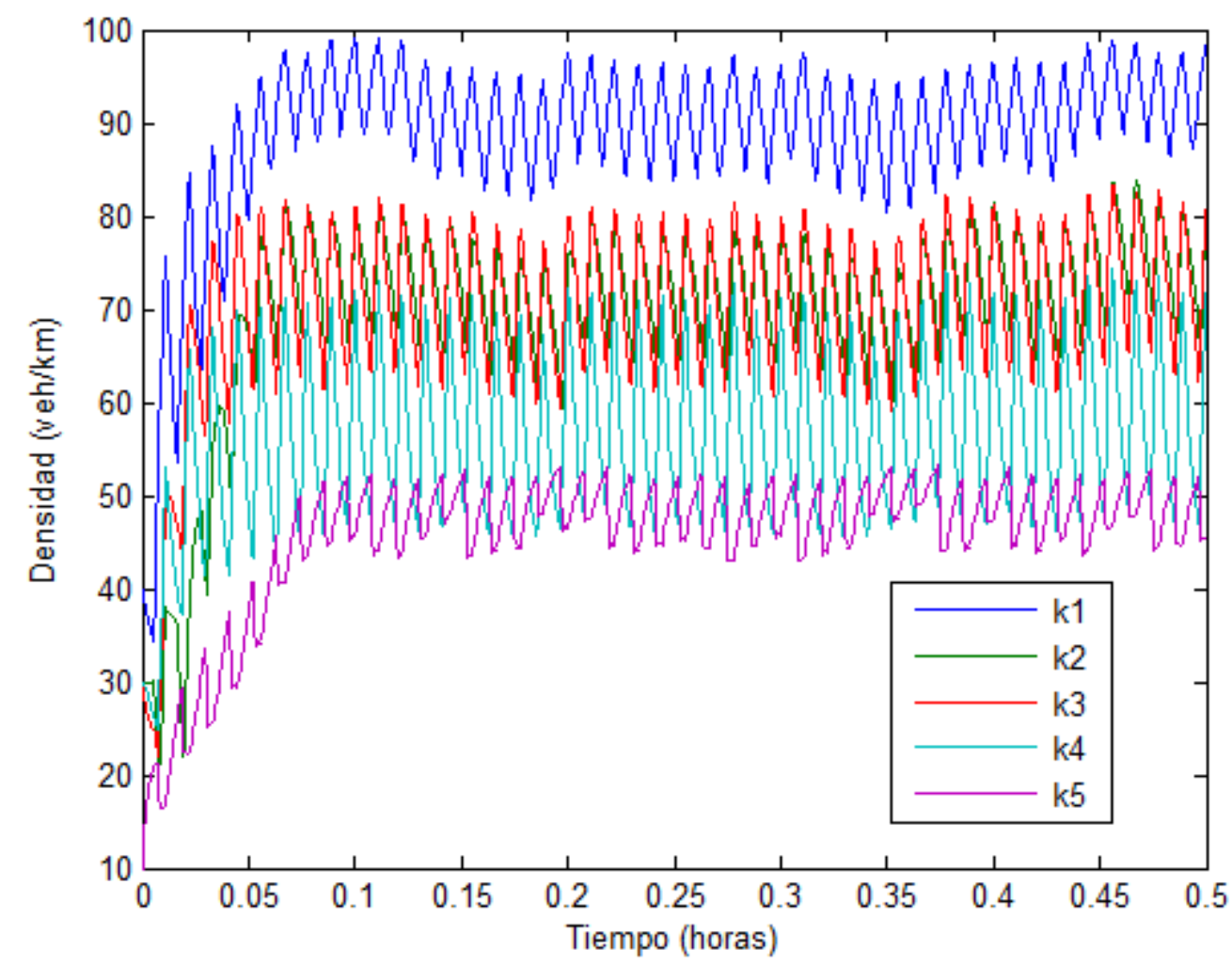

Figura 9 - Densidad en cada cuadra para la configuración óptima. 


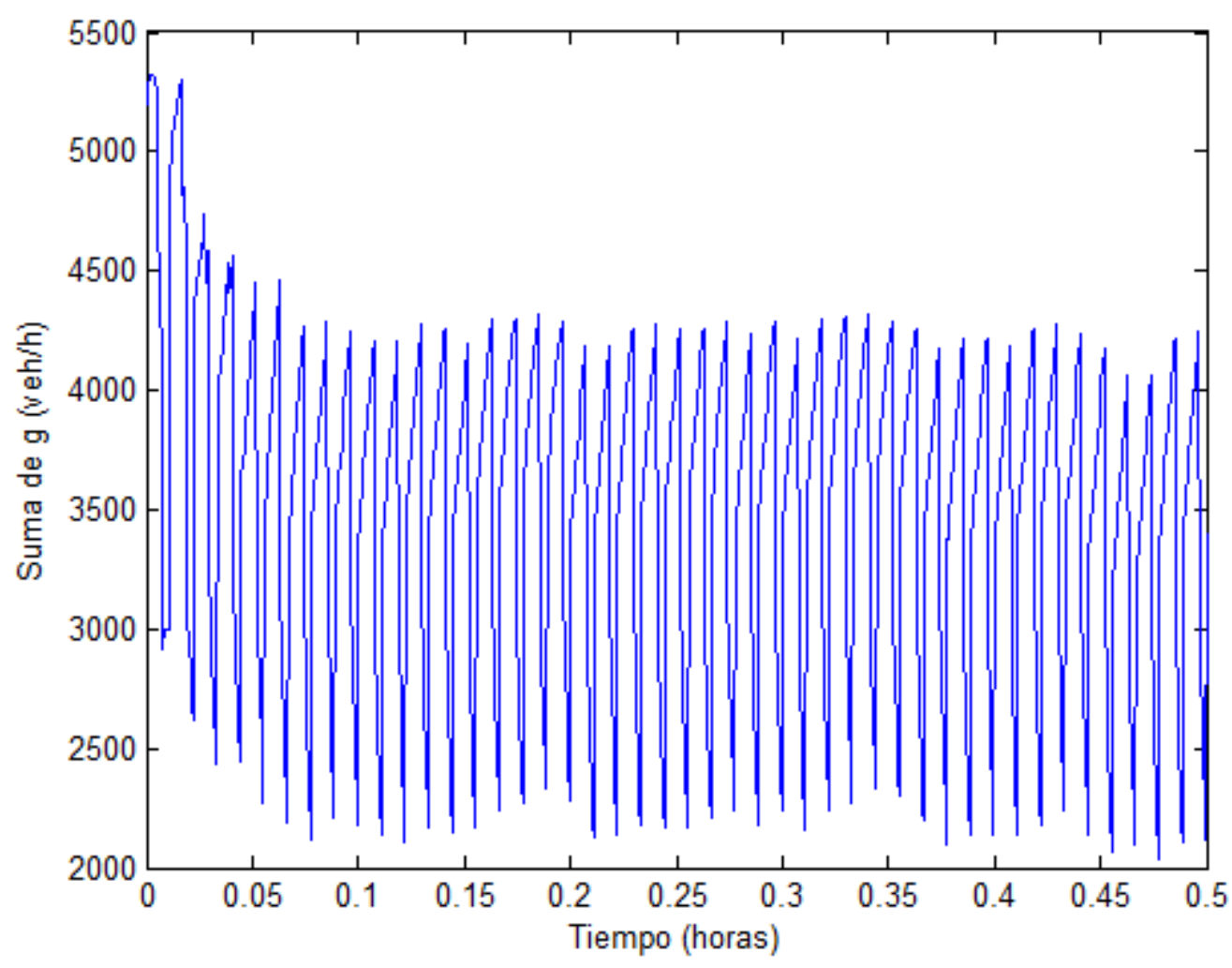

Figura 10 - Suma de flujos salientes de tramos 1 a7, 10, 11 y 14 para la configuración óptima.

\section{REFERENCIAS}

ABU-LEBDEH, G. y BANCHOAL, R. (1997). Development of a traffic control and queue management procedure for oversaturated arterials. Transportation Research Board Annual Meeting. Paper\#970707.

COCLITE, G., GARAVELLO, M., PICCOLI, B. (2005). Traffic flow on a road network. SIAM Journal on Mathematical Analysis 36 (6), pp. 1862-1886.

COURAGE, K y WALLACE, C. (1991). TRANSYT-7F User's Guide. Federal Highway Administration.

DAGANZO, C.F. (1994). The cell transmission model: A dynamic representation of highway traffic consistent with the hydrodynamic theory. Transportation Research Part B 28 (4). pp. 269-287.

DAGANZO, C.F. (1995). The cell transmission model, Part II: Network traffic. Transportation Research Part B 29 (2). pp. 79-93.

DOMINGUEZ, P.N y CORTÍNEZ, V.H. (2012). Un método continuo-discreto para el diseño óptimo de sistemas de transporte urbano. Mecánica Computacional XXXI, pp. 33353355. http://www.cimec.org.ar/ojs/index.php/mc

GAZIS, D. y POTTS, R. (1963) The oversaturated intersection. In 2nd International Symposium on TrafficTheory, ppp. 221-237. London, UK.

GIPPS, P.G. (1986), Nonlinear follow-the-lider models of traffic flow. Operations Research 9 (4), pp. 545-567. 
GÖTTLICH, S., HERTY, M. y ZIEGLER, U. (2015). Modeling and optimizing traffic light settings on road networks. Computers \& Operations Research 55, pp. 36-51.

GREENSHIELDS, B.D., (1934) A study of traffic capacity. Proceedings of the Highway Research Board 14, pp. 448-477.

HERMAN, R.C, MONTROLL, E.W., POTTS, R.B, ROTHERY, R.W. (1959). Traffic dynamics: Analysis of stability in car-following”. Operations Research 7, pp. 86-106.

JIN, W.L. (2013). A link queue model of network traffic flow. arXiv preprint arXiv:1209.2361.

LIGHTHILL, M.J. y WHITHAM, G.B. (1955). On kinematic waves: II. A theory of traffic flow on long crowded roads. Proceedings of the Royal Society of London A 229 (1178), pp. 317-345.

LO, H.K., CHANG, E., CHAN, Y.C. (2001). Dynamic network traffic control. Transportation Research Part A 35, pp. 721-744.

MAYORANO, F.J., RUBIALES, A.J., BORONI, G.A., BHOURI, N. Y LOTITO, P.A. (2007). Control óptimo del tráfico mediante semáforos con observadores locales. Mecánica Computacional XXIV, pp. 3343-3358. http://www.cimec.org.ar/ojs/index.php/mc

NAGEL, k. y SCHRECKENBERG, M. (1992). A cellular automaton model for freeway traffic. Journal de Physique I France 2 (2), pp. 2221-2229.

RICHARDS, P.I. (1956). Shock wave on the highway. Operations Research 4 (1), pp. $42-$ 51. 